\title{
Value-Based Software Traceability Workshop (VALSOT 2011)
}

\author{
Angelina Espinoza ${ }^{1}$, Richard Paige ${ }^{2}$, and Juan Garbajosa ${ }^{1}$ \\ 1 Technical University of Madrid (UPM), \\ E.U. Informatica, Spain \\ aespinoza@syst.eui.upm.es, jgs@eui.upm.es \\ http://syst.eui.upm.es \\ 2 University of York, Department of Computer Science, UK \\ paige@cs.york.ac.uk \\ http://www.cs.york.ac.uk/ paige
}

Traceability defines and maintains relationships among the artifacts involved in the software-engineering life cycle. This mechanism is widely used for purposes as project visibility and accountability, and it provides an essential support for developing high-quality software systems. Traceability has traditionally been strongly influenced by software process drivers; however, during the last few years, the software process is starting to take into account new drivers. One such driver is the software product's value. The global value of a product can be understood as the perceived benefits in relation to its cost. Both business and technical areas of an organization consider the value concept as a priority, for analyzing software costs and benefits. For these purposes, a tight interaction between these two sides is fundamental.

From the perspective of traceability, the value consideration may change many assumptions. For instance, the development artifacts that are involved in traceability (requirement, user story, design items, test unit, etc) should contain the necessary information to estimate its value under a given context. Similarly, traceability items (tracing links, tasks or resources to support traceability) need also to consider the value concept. Thereafter, the traditional traceability schemes seem to be too limited for describing and tracing the value. Since, business areas are involved and interested in software value tracing, the traceability approaches need to be more streamlined than those ones more technical oriented. Additionally, the agile community advocates the advantages of lean traceability for avoiding bothersome tasks that are difficult to perform in agile contexts.

The workshop aims to provide the attendees with an opportunity to develop a common research agenda through paper presentations, brainstorming, discussion and building consensus on important directions. As outcome, a report will be produced clearly outlining 1) the research topics and challenges in terms of specific problems in the area, and (2) a summary of existing solutions for targeting these topics, based on industrial experiences and academic pilots. Finally, a main expected result is to share among participants proved approaches in organizations. The aim is to spread useful traceability schemes for value-based product management, and for efficiently supporting agile projects. 